Proceedings of the Creative Construction Conference (2018)

Edited by: Miroslaw J. Skibniewski \& Miklos Hajdu

DOI 10.3311/CCC2018-035

Creative Construction Conference 2018, CCC 2018, 30 June - 3 July 2018, Ljubljana, Slovenia

\title{
A Study on Adjustment Method of Finishing Work Schedule based on Image Detection for High-Rise Building Construction
}

\author{
Taekyu Ko ${ }^{\mathrm{a}}$, Joonghwan Shin ${ }^{\mathrm{a}}$, Soonwook Kwon ${ }^{\mathrm{b}}$, Callejas-Sandoval, S. ${ }^{\mathrm{a}}$, Heesung Park ${ }^{\mathrm{c}}$, \\ Jaewoo Park \\ ${ }^{a}$ Department of Convergence Engineering for Future City, Graduate School, Sungkyunkwan University, 2066, Seoburo, Jangan-gu, Suwon-si, \\ Gyeonggi-do, Republic of Korea \\ ${ }^{b}$ School of Civil \& Architectural Engineering, and Landscape Architecture, Sungkyunkwan University, 2066, Seoburo, Jangan-gu, Suwon-si, \\ Gyeonggi-do, Republic of Korea \\ ${ }^{c}$ Deptartment of Civil \&Environmental Engineering, Hanbat National University, 125, Dongseo-daero, Yuseong-gu, Daejeon, Republic of Korea \\ *Construction Policy Research Institute, Korea Institute of Construction Technology, Goyang-daero, Goyang-si, Gyeonggi-do, 10223, Republic of \\ Korea
}

\begin{abstract}
As recent buildings have been getting higher and larger, the finishing work management has become a key factor for successful projects. Although many finishing work management techniques have been trying to set up a measureable scheduling plan at early stages before construction lift installation, but schedule adjustments according to site condition and construction progress are inevitable because there are many unexpected variables. To develop the assistance tool for finishing work management techniques such as TACT or LOB(Line of balance), in this study, we suggested image-detection(helmet detection) based labor counting method. The internet protocol cameras at each inside of construction lift cages collect and send photography data to processing server. And through image detection processing using photography data, it is possible to count the number of workers put into each finishing work. The worker count result is used for comparison with the existing finishing work schedule. This study has served as a key lead of lift monitoring based construction process management. Therefore, it is anticipated to settle as a system based data-centric construction management technique in the field.
\end{abstract}

(C) 2018 The Authors. Published by Diamond Congress Ltd., Budapest University of Technology and Economics Peer-review under responsibility of the scientific committee of the Creative Construction Conference 2018.

Keywords: Constucruction Lift, Finishing work schedule, Image Detection, Helmet detection

\section{Introduction}

Over the past few years, the number of high-rise buildings constructed in Korea has significantly increased. In case of Korea, there are many buildings over 500m high, which completed in 2017. Despite the development of high-rise construction, there are still many problems on process management. Especially, there are many needs of finishing work improvement caused by mix of work activities at the same time and the rise of vertical movement distance.

Finishing work takes part of approximately $15 \sim 20 \%$ of total construction cost. As buildings get higher, the most critical problem of finishing work phase is lifting management regarding manpower and materials [1]. The main reasons for lifting management problems are caused by long distance vertical transportation and increase of materials on finishing work. In case of high-rise building over 500m, in the Republic of Korea, an additional cost of over \$ 20 million is generated because of finishing work delays over 3 months. Such kinds of additional cost, incurred frequently, show the problem of current lifting plan which is used in most of high-rise construction projects.

Corresponding author: Author email: jwpark@kict.re.kr 
The existing method on finishing work schedule management, including lifting plan for each construction phase and peak-time period, is based on labor productivity $\left(\mathrm{Man} \cdot \mathrm{Day} / \mathrm{m}^{2}\right)$ which has been generally used in buildings under $300 \mathrm{~m}$ high. Therefore, another access to correct errors of existing lift management is requested for high-rise building construction.

\section{Finishing work schedule management for high-rise construction}

Although there are differences in each company and in each region, the TACT technique is most commonly used for finishing work management for high-rise building construction [2]. The TACT technique is a finishing work process management method for sub-dividing work into each floor and process, distributing human resources and equipment into even periods of each construction time, adjusting the preceding and lagging relationship of works on the same floor, and planning for the sequential operation of the work on each floor. Even though we are not able to establish whether every vertical plan is based on the TACT technique, there is no theoretical difference between the as-is vertical plan and TACT.

\begin{tabular}{|c|c|c|}
\hline $\begin{array}{c}\text { Finishing Work } \\
\text { Activity }\end{array}$ & Man:Day/ $\mathrm{m}^{2}$ & $\mathrm{~m}^{2} /$ Man:Day \\
\hline Activity 1 & $\mathrm{X} 1$ & $1 / \mathrm{X} 1$ \\
\hline Activity 2 & $\mathrm{X} 2$ & $1 / \mathrm{X} 2$ \\
\hline Activity & $\mathrm{X} 3$ & $1 / \mathrm{X} 3$ \\
\hline Activity & $\mathrm{X} 4$ & $1 / \mathrm{X} 4$ \\
\hline$\vdots$ & $\vdots$ & $\vdots$ \\
\hline
\end{tabular}

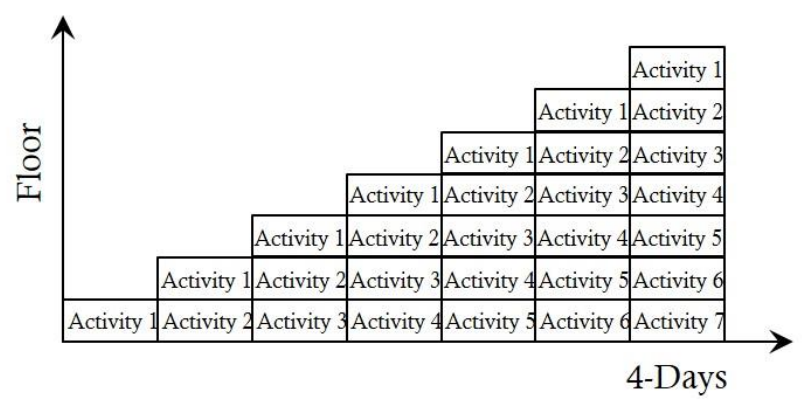

Fig. 1. TACT based Work Activity Arrangement

TACT is performed using the process schedule, assuming that all the TACT-elements are implemented in the same manner within the consistent cycle. However, the degree of progress in TACT process plans is subject to be changed depending on the work situations in the field. In addition, TACT makes it feasible to find delay factors and modify TACT on the plan, using analytic data of the operation and change plan to suit the current situation.

The number of mobile workers on each floor is a very important measure as a basis for judging the progress of the current finishing work. The field manager can check the progress of each finishing activity by monitoring the number of workers on a daily basis from 7:00 to 8:00 (the usual time the worker is assigned to each floor).

\section{Unmanned Construction Lift \& Sensor based Monitoring}

In addition to counting the number of workers, various lift sensors are essential to monitor lift conditions for finishing work. Especially, 'position', 'moving direction', 'call floor' and 'target floor' are important factors in recognizing the worker's assigned target. The sensor module consist of Double Senor type Encoder, Limit Switch, Separating type Current Transformer, Proximity Sensor, Load Cell. The processing board of sensor data at inside of construction lift cage and encoder sensor are as shown in Figure 2 and Figure 3.

This study has expended the sensor-based monitoring from the lift operation with a driver, to the unmanned operation system. The main difference of sensor monitoring between existing manual operation system and unmanned system is the target floor input board. Because the unmanned system does not have a driver, the workers have to appoint a lift moving target themselves. By worker's choice, lift-car's estimated moving distance and time are calculated according to height of floor which pre-set in operation processing board and cycle-time equation as shown in (1). 


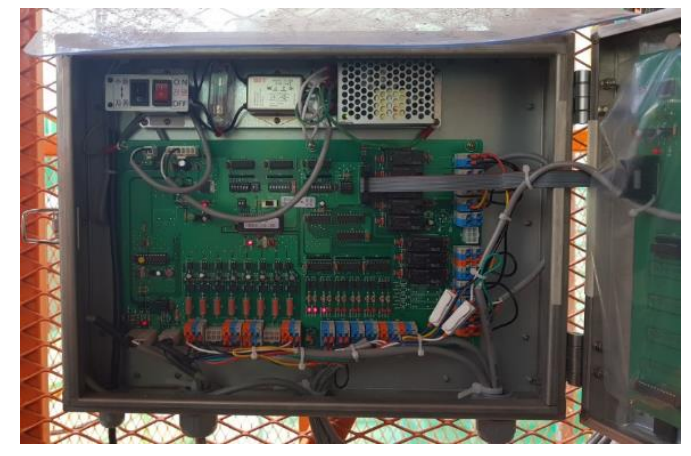

Fig. 4. Data Processing board

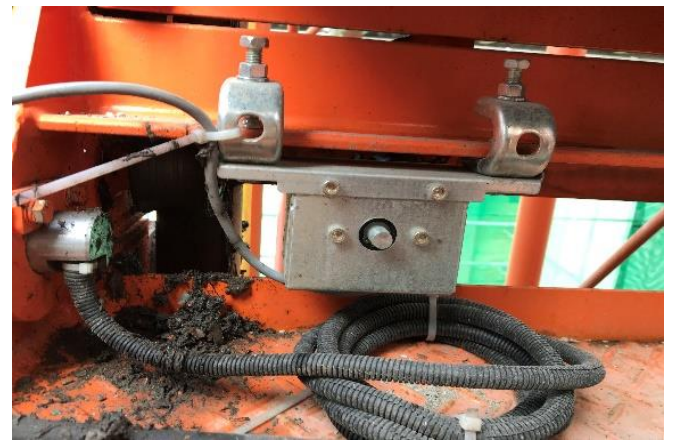

Fig. 5. Encoder Sensor

$$
\text { Unit Cycles }=t_{0}+\left(s_{r} \times t_{1}\right)+2\left\{\left(\left(s_{r}+1\right) \times t_{a}\right)+\left\{\left(h_{r}-h_{r-1}\right)-\left(\left(s_{r}+1\right) \times d_{a}\right)\right\} / v\right\}
$$

The unit-cycle time equation includes loading time $\left(t_{0}\right)$, unloading time $\left(t_{1}\right)$, number of stops $\left(s_{r}\right)$, height of stop floor $\left(h_{r}\right)$, accelerating/decelerating time $\left(t_{a}, d_{a}\right)$ and maximum lift speed(v). Eq. (1) can be used to determine the number of workers for each floor by matching movement history data from encoder and Image detection data. The unmanned lift system supports to make data matching faster. Figure 4 and 5 shows the components of the unmanned construction lift system including calling device and target floor input board.

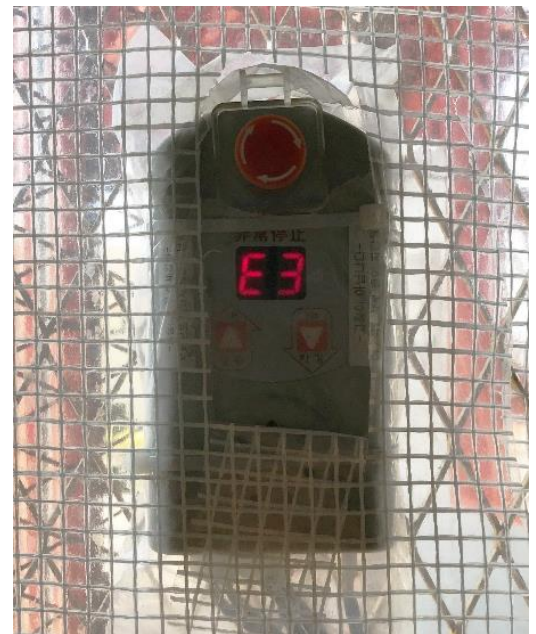

Fig. 4. Calling Device

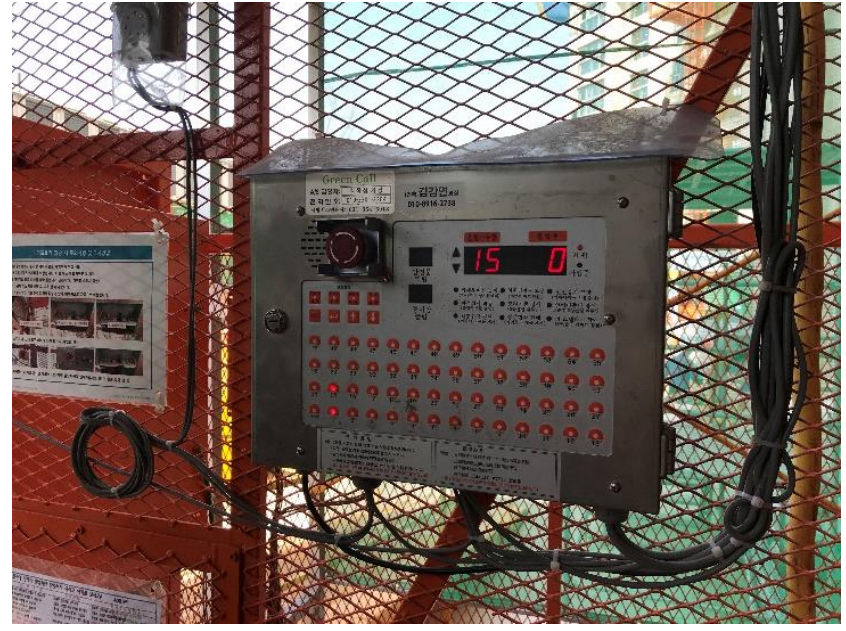

Fig. 5. Target floor input board

\section{How to count man power}

- It is necessary to look at various image-detection and deep-learning technologies as open source considering the environment inside the construction lift-car, where frequent visibility redundancy occurs. Deep-learning based computer vision is the core technology to record object state changes inside the lift-car.

\subsection{Selection of image detection method}

To figure out most suitable object detection method, we conducted comparative reviews in terms of performance and objectives of various commercial products and research. The comparison of each image-detection is as shown in Table 1. 
Table 1. An example of object detection methods.

\begin{tabular}{ll}
\hline Examples & Characteristics \\
\hline R-CNN & $\begin{array}{l}\text { Testing time per image 50 seconds. The mean average precision } \\
\text { (mAP) for each query is of 66\% on VOC 2007 test-dev. [5] }\end{array}$ \\
It builds on previous work to efficiently classify object \\
proposals using deep convolutional networks. Achieves near \\
real-time rates using very deep networks, at 2s per image. Multi- \\
scale design based on anchors, computed on a single-scale \\
image. [7] \\
It is a single, unified network for object detection. It does not \\
need external region proposal. The test-time speed of obtaining \\
results is of 200ms per image. [5] \\
It processes images in real-time at 45 frames per second. On a \\
Pascal Titan X it processes images at 40-90 FPS. \\
You Only Look Once (YOLO) \\
The SSD approach is based on a feed-forward convolutional \\
network that produces a fixed-size collection of bounding boxes \\
and scores for the presence of object class instances in those \\
boxes, followed by a non-maximum suppression step to produce \\
the final detections. [6]
\end{tabular}

Object detection methods perform different from one another, as shown in Table 1. This section describes the different accuracy on real-time detection, the approach applied to stage detectors bounding boxes and applying highquality classifiers. R-CNN applies the CNN feature extraction to possible objects and then finally classifies the regions. In comparison, Fast R-CNN used a single network to compute image features (CNN), classify (SVM), and tighten bounding boxes (regressor) [7]. Subsequently, in Faster R-CNN, classification and a single CNN is used for region proposals, instead of using the special region proposal method used in Fast R-CNN [5]. Similarly, YOLO proposed a new approach to object detection using a simple convolutional neural network, allowing to process images in real-time at 45 frames per second. Finally, SDD has comparable accuracy to methods that utilize an additional object proposal step, providing a unified framework for both training and inference. [6]

\subsection{YOLO based Detection System}

Considering the characteristics of the lift-car at which the internal conditions change from time to time, the accuracy of processing results, and the speed, we selected the YOLO(You Only Look Once) as the image detection technology. The image detection procedure using YOLO for our system is as follows [8].

(1) Generate image flame group sampling for analysis

(2) Runs a single convolutional network on the image

(3) Thresholds the resulting detections by the model's confidence

(4) Moves the frame group and repeats the processing analysis

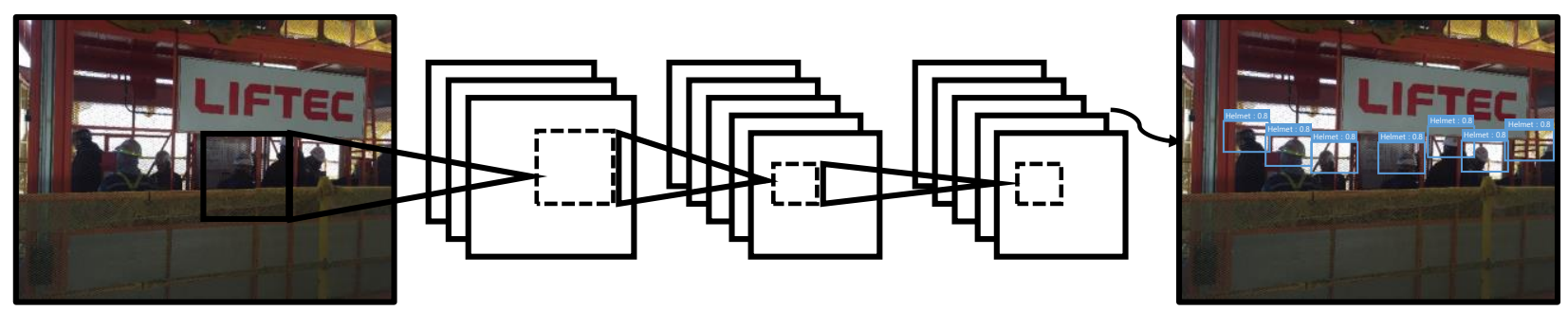

Fig. 6. YOLO Detection System

As shown in Fig. 6, the bounding box at each convolution network step calculate the reliability for the image. In initial step, the reliability of image can be low; however, by adjusting the position of the bounding box-the accuracy of object recognition can be improved. The YOLO-based image detection method has the following advantages through correction through repetitive frame movement. 
First, it can derive result faster since regression problem is used as detection method.

Second, comparing to other detection methods, the number of background errors rarely occurs.

Third, YOLO is highly generalizable, it is less likely to break down when applied to new domains or unexpected inputs.

\subsection{Counting Manpower on the lift}

The target for image detection is a helmet, to count the number of workers on the construction lift and the number of workers aboard each floor. Because all person on the construction lift wear the safety helmet, the helmet-targeted counting is more effective than counting the shape of a person.
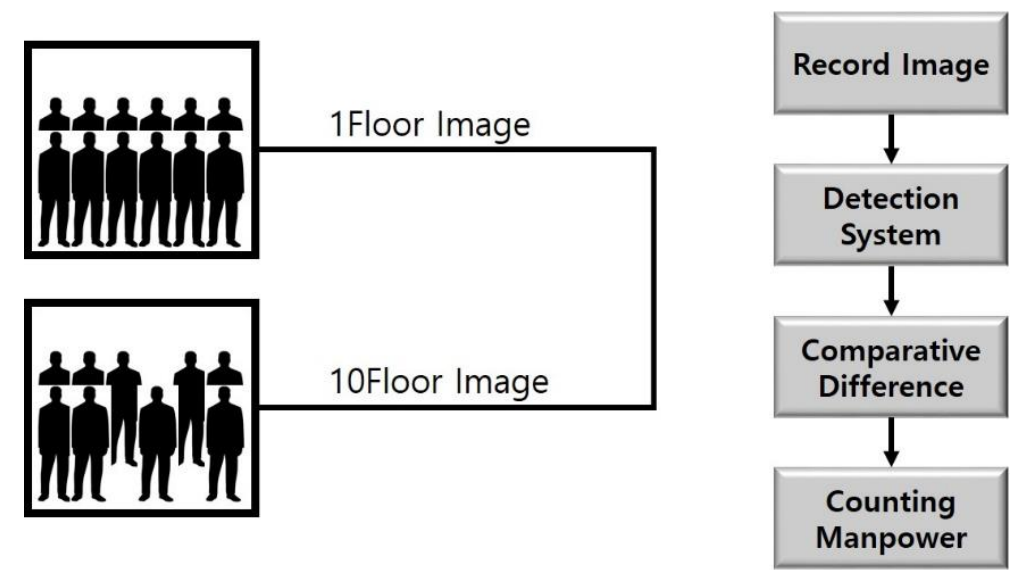

Fig. 7. Image Detection based Counting Method

As shown in Fig. 7, by comparing the number of passengers at the start of the lift and the status of the passengers after getting off a certain floor, it is possible to verify the number of passenger that got off in the floor. According to image detection based comparison, the data of how many workers have left at each floor could be collected and make lifting history of workers.

\section{Adjustment Method of Finishing Work Schedule based on Image Detection}

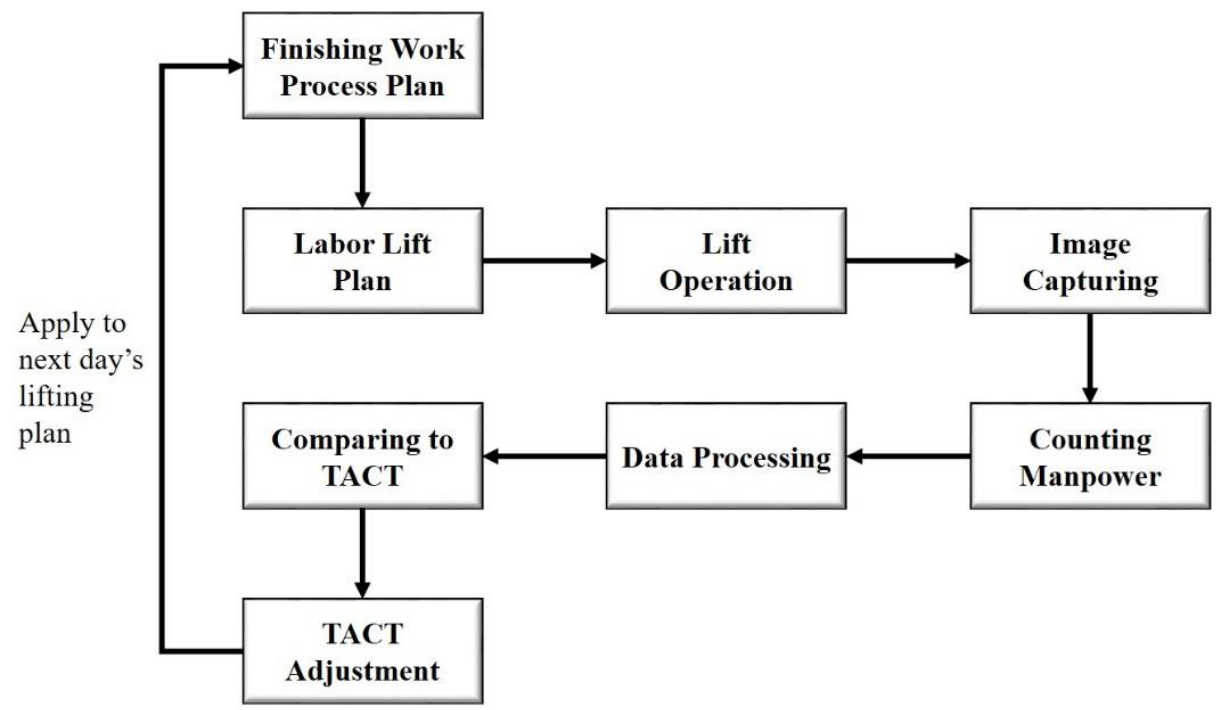

Fig. 8. TACT Adjustment Procedure 
As shown in Fig. 8, it is possible to move to the planning stage with the existing planned TACT plan by composing the history of the getting off person from each floor. Because the TACT plan is based on the expected worker productivity for each task activity, the field manager can compare the plan and actual view from the short - term and long - term perspective by comparing the number of counting workers.

From a long-term perspective, the field manager can determine and correct whether the projected worker productivity is appropriate for the site, and from a short-term point of view, whether the work activity is delayed on the TACT process. If there is a delay in a particular activity, the field manager can adjust the time span by adjusting the TACT schedule.

In the short term, matching between image detection based labor management and TACT schedule is performed through the following procedure.

First, according to the planned process plan, the expected worker is placed on field.

Second, we collect and analyze information through lift manpower transport. And we acquire movement patterns of number of worker.

Third, TACT time is adjusted based on the expected schedule of the TACT process and the moving pattern of the actual number of worker.

Fourth, efficient manpower management is possible through the application of adjusted TACT time to the next day's process plan

\section{Conclusion}

As the buildings became high-risen, and the limits on the maximum lifted floors increased, variables applied to the management of finalizing construction became complicated. Hereupon, operation management of the lift is needed for the construction after implementing finalizing process along with a decision-making procedure on the transportcirculation of finishing work materials that are appropriate for flexibly changed situations in the field.

Therefore, in this study, operation history information by ip-camera was saved as image detection result. Then it was utilized to draw number of workers for each finishing work. Based on image detection technology and TACT technique, we proposed correction method to finishing work process management. Lastly, this study has served as a starting point of vision technology based lift-car management system. Therefore, it is anticipated to settle as a system based on unmanned smart lift through verification made in the entire period of research project.

\section{Acknowledgement.}

This research was supported by a grant(18AUDP-B106327-04) from Architecture \& Urban Development Research Program funded by Ministry of Land, Infrastructure and Transport of Korean government.

This work is financially supported by Korea Ministry of Land, Infrastructure and Transport(MOLIT) as Smart City Master and Doctor Course Grant Program..

\section{References}

[1] R. Sacks, R. Navon, I. Brodetskaia and A. Shapira, Feasibility of Automated Monitoring of Lifting Equipment in Support of Project Control, J. Constr. Eng. Manage, 2005, pp. 604-614

[2] C.Y. Cho, Y.S. Shin, S.K. Kim, J.Y. Kim and M.Y. Cho, An Algorithm for Hoisting Time Calculation in Super-tall Building Construction, Korean journal of Construction Engineering and Management, 2011, pp. 120-129

[3] S.K. Kim, K.K. Han, A Study on the Estimation of Proper Number of Construction Lifts, The Korea Institute of Building Construction, 2008, pp. $119-125$

[4] Y.S. Shin, Construction Lift Planning System in Tall Building Construction, Architectural Institute Korea, 2011, pp. 227-235

[5] S, Ren, Faster R-CNN: Towards Real-Time Object Detection with Region Proposal Networks, Retrieved from journal, 2016

[6] W. Liu, D. Anguelov, D. Erhan, C. Szegedy, and S. Reed. SSD: Single shot multibox detector. In ECCV, 2016

[7] R. Girshick, Fast R-CNN, Retrieved from journal, 2015

[8] S. Redmon, S. Divvala, R. Girshick, A. Farhadi, You Only Look Once: Unified, Real-Time Object Detection. Retrieved from journal, 2016, pp. 779-788 